\title{
Solving the Inverse Problems of Wave Equation by a Boundary Functional Method
}

\author{
Chein-Shan Liu ${ }^{1,2}$ and Yung-Wei Chen ${ }^{3}$ \\ 1. Center for Numerical Simulation Software in Engineering and Sciences, College of Mechanics and Materials, Hohai University, \\ Jiangsu 210098, China \\ 2. Department of Mechanical and Mechatronic Engineering, National Taiwan Ocean University, Keelung 20224, Taiwan \\ 3. Department of Marine Engineering, National Taiwan Ocean University, Keelung 20224, Taiwan
}

\begin{abstract}
The inverse problems of wave equation to recover unknown space-time dependent functions of wave speed and wave source are solved in this paper, without needing of initial conditions and no internal measurement of data being required. After a homogenization technique, a sequence of spatial boundary functions at least the fourth-order polynomials are derived, which satisfy the homogeneous boundary conditions. The boundary functions and the zero element constitute a linear space, and then a new boundary functional is proved in the linear space, of which the energy is preserved for each dynamic energetic boundary function. The linear systems and iterative algorithms used to recover unknown wave speed and wave source functions with the dynamic energetic boundary functions as bases are developed, which converge fast at each time step. The input data are parsimonious, merely the measured boundary strains and the boundary values and slopes of unknown functions to be recovered. The accuracy and robustness of present methods are confirmed by comparing exact solutions with estimated results under large noises up to $20 \%$.
\end{abstract}

Key words: Space-time dependent wave speed, space-time dependent wave source, dynamic energetic boundary functions, BFM (boundary functional method), iterative method.

\section{Introduction}

Studying the wave motions is a very important topic for many physical and engineering problems, for instance the stress wave in solids, the wave propagation in fluids, the scattering problems of electromagnetic waves, and the sound wave propagation in media. For the direct problems of wave equations there are many available methods and the techniques are matured; for example, Lin et al. [1] have developed a fast solver of the three-dimensional wave equation by using the sparse scheme of the method of fundamental solutions, and Liu et al. [2] have developed a multiple-direction Trefftz method for solving the three-dimensional wave equation in arbitrary spatial domain.

In contrast, the parameter characterizing of material

Corresponding author: Yung-Wei Chen, Ph.D., associate professor, research field: inverse problem. property distributed in a solid medium may be quite complicated, which depends on time and position. The inverse scattering technique is how we can obtain a large part of our information about the constituent. We know about the interior structure of the earth by solving the inverse problem of determining the sound speed by measuring the travel times of seismic waves. The inverse scattering technique is also used in the non-destructive testing of structures to find cracks and corrosion.

For the problems of material property identification of wave equations there are two kinds of approaches: time-independent approach and time-dependent approach. In fact, most often the inverse scattering problems are stated in a time-independent formulation after taking a Fourier transformation in the time variable, or inserting a time-harmonic plane wave into the wave equations. However, when the material is time aging, such as the viscoelastic material, whose 
coefficient may be time-dependent, and hence the time-independent approach is not valid for the identification of time-dependent material coefficient. In the time-domain approach of the inverse scattering problems, there is some literature related to the present issue, to name a few, Baev [3-5], Tadi [6-8], and Na and Kallivokas [9].

The parameter identification in partial differential equations from over-specified data is widely encountered in the modelling of physical phenomena. First, we consider an inverse coefficient problem to recover an unknown parameter $\alpha(x, t)$ in a one-dimensional wave equation, of which one needs to find displacement $u(x, t)$ as well as the wave speed function $\alpha(x, t)$ that simultaneously satisfy:

$$
\begin{gathered}
\frac{\partial^{2} u(x, t)}{\partial t^{2}}=\frac{\partial}{\partial x}\left[\alpha(x, t) \frac{\partial u(x, t)}{\partial x}\right]+H(x, t) \\
0<x<\ell, \quad 0<t \leq t_{f}
\end{gathered}
$$

where, $H(x, t)$ is a given source function. For Eq. (1), there is another inverse problem to identify the wave source function $H(x, t)$, which is the next issue to be solved in this paper. When both the inverse problems are solved without providing internal measurements of data and initial conditions, none is a simple and easily solved inverse problem.

Because the above problem has an unknown function $\alpha(x, t)$ and gives no initial conditions, it cannot be solved directly to find $u(x, t)$. In order to recover $\alpha(x, t)$ we give over-specified data of

$$
\begin{gathered}
u(0, t)=F_{0}(t), \quad u(\ell, t)=F_{\ell}(t), \\
u_{x}(0, t)=Q_{0}(t), \quad u_{x}(\ell, t)=Q_{\ell}(t) \\
\alpha(0, t)=\alpha_{0}(t), \quad \alpha(\ell, t)=\alpha_{\ell}(t), \\
\alpha^{\prime}(0, t)=\alpha_{0}^{\prime}(t), \quad \alpha^{\prime}(\ell, t)=\alpha_{\ell}^{\prime}(t)
\end{gathered}
$$

where, $F_{0}(t)$ and $F_{\ell}(t)$ are respectively the given functions of left-boundary value and right-boundary value of $u(x, t)$, and $Q_{0}(t)$ and $Q_{\ell}(t)$ are respectively the given strains on left-boundary and right-boundary. It can be seen that the extra data required to recover $\alpha(x, t)$ are parsimonious, merely the measured boundary strains and the boundary values and slopes of unknown functions to be recovered.

The inverse problems are those in which one would like to determine the causes for an observed effect, which are usually ill-posed. For the present inverse problem, the observed effect is the boundary strains. We are interested to search the unknown coefficient $\alpha(x, t)$ in Eq. (1), which causes the effect we observe through the measurement of boundary strains in time.

However, using Eqs. (1)-(3) to recover the unknown space-time wave speed $\alpha(x, t)$ is a very difficult task, because the system in Eqs. (1)-(3) is seriously under-determined, and the resulting inverse problem is severely ill-posed. For this inverse wave speed problem, the measurement error may lead to a large discrepancy from the true cause. The numerical methods for solving the wave speed identification problems have been examined by many researchers, for example Refs. [10-15]. Note that we do not need the data from the initial displacement and initial velocity, which are drastically different from that in the above numerical methods.

It is known that the wave equation constitutes a hyperbolic system, of which there are some works on the identifications of the point sources [16-20]. This study has important application in the seismology detection, which could be regarded as an approximation of elastic waves generated from the point dislocation. The excitation force is assumed to have known time profile, and the problem is to determine the spatial variation from supplementary measurements.

Here we attempt to accurately and quickly identify the wave speed function and wave source function sequentially in time by solving the inverse wave propagation problems without needing of initial conditions, final time displacement, and internal 
measurements of displacement data. This identification technique can find some wide range applications in engineering and science, since it is often easier to measure the boundary strains in time, rather than that to directly measure the displacement inside the propagating material. Due to its importance on the knowledge to the wave propagation properties and wave sources for new materials used in many system analyses, these inverse problems have attracted much attention.

The remainder of this paper is arranged as follows. In Section 2 we introduce a new idea of the boundary functional in terms of energetic boundary functions, which constitute a linear space of all polynomial functions with at least the fourth-order, to automatically satisfy the homogeneous boundary conditions. In Section 3 we derive the iterative algorithm to recover the unknown wave speed functions and four examples are given in Section 4. Then, we derive the iterative algorithm to recover the unknown space-time dependent wave source functions in Section 5, where three numerical examples are given. Finally, the conclusions are drawn in Section 6.

\section{Dynamic Energetic Functional of Boundary Functions}

Multiplying both sides of Eq. (1) by $u(x, t)$, we have:

$$
\frac{\partial^{2} u(x, t)}{\partial t^{2}} u(x, t)=\frac{\partial}{\partial x}\left[\alpha(x, t) \frac{\partial u(x, t)}{\partial x}\right] u(x, t)+H(x, t) u(x, t)
$$

Integrating it from $x=0$ to $x=\ell$ and using the integration by parts one time we can derive

$$
\begin{array}{r}
\int_{0}^{\ell} u(x, t) \ddot{u}(x, t) d x+\int_{0}^{\ell} \alpha(x, t) u^{\prime}(x, t)^{2} d x-\int_{0}^{\ell} H(x, t) u(x, t) d x \\
=\alpha_{\ell}(t) Q_{\ell}(t) F_{\ell}(t)-\alpha_{0}(t) Q_{0}(t) F_{0}(t):=F(t)
\end{array}
$$

where, the boundary conditions in Eqs. (2) and (3) were used. The above the superimposed dot denotes the time differential, while a prime denotes the spatial differential. The resulting equation is a dynamic energy equation due to its time-dependence. This encourages us to use the energy functional as a mathematical tool to identify $\alpha(x, t)$.

Usually, the given data in Eq. (2) are not zero, which leaves us an obstacle to set up a linear space to be introduced below. Before embarking the analysis of wave speed we seek a variable transformation by

$$
v(x, t)=u(x, t)-B_{0}(x, t)
$$

where,

$$
\begin{aligned}
B_{0}(x, t)=\frac{1}{\ell^{3}}\left[2 F_{0}(t)-2 F_{\ell}(t)\right. & \left.+Q_{0}(t) \ell+Q_{\ell}(t) \ell\right] x^{3}-\frac{1}{\ell^{2}}\left[3 F_{0}(t)-3 F_{\ell}(t)+2 Q_{0}(t) \ell+Q_{\ell}(t) \ell\right] x^{2} \\
& +Q_{0}(t) x+F_{0}(t)
\end{aligned}
$$

is a homogenization function, such that we have a new energy functional in terms of $v(x, t)$ with the homogeneous boundary conditions for $v(x, t)$ :

$$
\begin{gathered}
\int_{0}^{\ell}\left[v(x, t)+B_{0}(x, t)\right]\left[\ddot{v}(x, t)+\ddot{B}_{0}(x, t)\right] d x+\int_{0}^{\ell} \alpha(x, t)\left[v^{\prime}(x, t)+B_{0}^{\prime}(x, t)\right]^{2} d x \\
-\int_{0}^{\ell} H(x, t)\left[v(x, t)+B_{0}(x, t)\right] d x=F(t) \\
v(0, t)=0, \quad v(\ell, t)=0, \quad v_{x}(0, t)=0, \quad v_{x}(\ell, t)=0
\end{gathered}
$$


We cannot exactly know $v(x, t)$ in Eq. (7), because $\alpha(x, t)$ is an unknown function to be determined. However, we can set up some functions to approximate $v(x, t)$. First, we can derive the boundary function which automatically satisfies the homogeneous boundary conditions in Eq. (8):

$$
B_{j}(x)=\left(x^{4}-2 \ell x^{3}+\ell^{2} x^{2}\right) x^{j-1}, \quad j \geq 1
$$

They are at least the fourth-order polynomial boundary functions, satisfying the following homogeneous boundary conditions:

$$
\begin{aligned}
& B_{j}(0)=0, B_{j}(\ell)=0 \\
& B_{j}^{\prime}(0)=0, B_{j}^{\prime}(\ell)=0
\end{aligned}
$$

From Eqs. (9) and (10) it is obvious that when $B_{j}(x)$ is a boundary function, $\beta B_{j}(\ell), \beta \in \mathbb{R}$ is also a boundary function, and when $B_{j}(x)$ and
$B_{k}(x)$ are boundary functions, $B_{j}(x)+B_{k}(x)$ is also a boundary function. The boundary functions are closure under a scalar multiplication and addition. So, the set of

$$
\left\{B_{j}(x)\right\}, j \geq 1
$$

and the zero element constitute a linear space of boundary functions, denoted by $B$.

The following result is important to help us identify the unknown wave speed function $\alpha(x, t)$.

Theorem 1 . In the linear space $B$ there exist homogeneous boundary functions:

$$
\begin{gathered}
E_{j}(x)=\gamma_{j} B_{j}(x)+B_{j+1}(x), \\
j \geq 1, j \text { not summed }
\end{gathered}
$$

such that $\alpha(x, t)$ is a solution of the following functional in terms of

$$
\int_{0}^{\ell}\left[E_{j}(x, t)+B_{0}(x, t)\right]\left[\ddot{B}_{0}(x, t)-H(x, t)\right] d x+\int_{0}^{\ell} \alpha(x, t)\left[E_{j}^{\prime}(x)+B_{0}^{\prime}(x, t)\right]^{2} d x=F(t),
$$

where,

$$
\begin{gathered}
a_{2}=\int_{0}^{\ell} \alpha(x, t) B_{j}^{\prime}(x)^{2} d x, \\
a_{1}=\int_{0}^{\ell}\left\{2 \alpha(x, t)\left[B_{j+1}^{\prime}+B_{0}^{\prime}(x, t)\right] B_{j}^{\prime}(x)+\left[\ddot{B}_{0}(x, t)-H(x, t)\right] B_{j}(x)\right\} d x, \\
a_{0}=\int_{0}^{\ell}\left\{\alpha(x, t)\left[B_{j+1}^{\prime}+B_{0}^{\prime}(x, t)\right]^{2}+\left[\ddot{B}_{0}(x, t)-H(x, t)\right]\left[B_{j+1}+B_{0}(x, t)\right]\right\} d x-F(t),
\end{gathered}
$$

$$
\gamma_{j}=\frac{-a_{1}-\sqrt{a_{1}^{2}-4 a_{0} a_{2}}}{2 a_{2}} .
$$

Proof. Because $B_{j}(x), B_{j+1}(x) \in B$ are elements in the linear space $B$, the linear combination in Eq. (12) renders $E_{j}(x) \in B$ an element in the linear space $B$, which satisfies the homogeneous boundary conditions:

$$
\begin{aligned}
& E_{j}(0)=0, E_{j}(\ell)=0, \\
& E_{j}^{\prime}(0)=0, E_{j}^{\prime}(\ell)=0
\end{aligned}
$$

due to Eq. (10).

Because $E_{j}(x)$ already satisfies the boundary conditions in Eq. (16), we turn our attention to the energy identity Eq. (7), from which we can approximate $v(x, t)$ by $E_{j}(x)$ and derive Eq. (13), which is a dynamic energetic functional of $E_{j}(x)$ defined in the linear space.

Inserting Eq. (12) for $E_{j}(x)$ and

$$
E_{j}^{\prime}(x)=\gamma_{j} B_{j}^{\prime}(x)+B_{j+1}^{\prime}(x)
$$

for $E_{j}^{\prime}(x)$ into Eq. (13) we can derive a quadratic equation for determining the multiplier $\gamma_{j}$ by

$$
a_{2} \gamma_{j}^{2}+a_{1} \gamma_{j}+a_{0}=0
$$

which is time-dependent due to $B_{0}(x, t)$ and $F(t)$, and the coefficients $a_{0}, a_{1}, a_{2}$ are defined in Eq. (14). Then the solution of $\gamma_{j}$ is derived in Eq. (15). This 
ends the proof of this theorem.

The above context, it seems unreasonable that we replace the function $v(x, t)$ of $(x, t)$ by a function $E_{j}(x)$ of $x$. Later, it will be clear that the function $E_{j}(x)$ is an implicit function of $t$ due to the dependence of $\gamma_{j}$ on $t$. The linear element $E_{j}(x)$ in Eq. (12) upon equipping with the above $\gamma_{j}$ is an implicit function of time and is a dynamic energetic boundary function, which not only satisfies the boundary conditions, but also preserves the dynamic energy in Eq. (13).

Up to here it is witnessed to determine the multiplier $\gamma_{j}$ by using the energy functional in Eq. (13). Due to this reason $E_{j}(x)$ is called a dynamic energetic boundary function, and correspondingly the numerical method based on $E_{j}(x)$ to be introduced below is a BFM (boundary functional method).

\section{Recovering the Wave Speed}

\subsection{Series Expansion of $\alpha(x, t)$}

Because the boundary data of $\alpha(x, t)$ are supplemented in Eq. (3), we can introduce the following translation function:

$$
\begin{gathered}
d(x, t)=\frac{1}{\ell^{3}}\left[2 \alpha_{0}(t)-2 \alpha_{\ell}(t)+\alpha_{0}^{\prime}(t) \ell+\alpha_{\ell}^{\prime}(t) \ell\right] x^{3} \\
-\frac{1}{\ell^{2}}\left[3 \alpha_{0}(t)-3 \alpha_{\ell}(t)+2 \alpha_{0}^{\prime}(t) \ell+\alpha_{\ell}^{\prime}(t) \ell\right] x^{2} \\
+\alpha_{0}^{\prime}(t) x+\alpha_{0}(t)
\end{gathered}
$$

such that we have

$$
\begin{aligned}
& d(0, t)=\alpha_{0}(t), d(\ell, t)=\alpha_{\ell}(t), \\
& d^{\prime}(0, t)=\alpha_{0}^{\prime}(t), d^{\prime}(\ell, t)=\alpha_{\ell}^{\prime}(t)
\end{aligned}
$$

We suppose that the unknown wave speed function $\alpha(x, t)$ can be expanded in terms of the bases functions $E_{k}(x)$ :

$$
\alpha(x, t)=d(x, t)+\sum_{k=1}^{m} b_{k} E_{k}(x),
$$

which automatically satisfies $\alpha(0, t)=\alpha_{0}(t)$, $\alpha(\ell, t)=\alpha_{\ell}(t) \quad, \quad \alpha^{\prime}(0, t)=\alpha_{0}^{\prime}(t) \quad$ and $\alpha^{\prime}(\ell, t)=\alpha_{\ell}^{\prime}(t)$, due to Eqs. (16) and (20).

Then, with an initial guess of $b_{k}$ we use the above $\alpha(x, t)$ to set up the linear elements system $E_{j}(x)$ by the method in Section 2. During the iteration process, $E_{j}(x)$ are modified by $\alpha(x, t)$ which is varying step-by-step.

We can derive a system of linear algebraic equations by inserting $\alpha(x, t)$ of Eq. (21) and different $E_{j}(x)$ with $j=1, \ldots, m$ into Eq. (13):

$$
\begin{aligned}
& b_{k} \int_{0}^{\ell}\left[E_{j}^{\prime}(x)+B_{0}^{\prime}(x, t)\right]^{2} E_{k}(x) d x \\
=F(t)+ & \int_{0}^{\ell}\left[H(x, t)-\ddot{B}_{0}(x, t)\right]\left[E_{j}(x)+B_{0}(x, t)\right] d x \\
& \quad-\int_{0}^{\ell} d(x, t)\left[E_{j}^{\prime}(x)+B_{0}^{\prime}(x, t)\right]^{2} d x .
\end{aligned}
$$

Solving this linear system we can determine the expansion coefficients $b_{k}, k=1, \ldots, m$. Then, we can estimate $\alpha(x, t)$ by Eq. (21).

\subsection{Iterative Algorithm to Recover $\alpha(x, t)$}

The numerical procedures of BFM are summarized as follows.

(i) Give $t$ in a time interval of $t \in\left(0, t_{f}\right]$, and give $m, \varepsilon$, and an initial guess of $\mathbf{b}^{\mathbf{0}}=\left(b_{1}, \ldots, b_{m}\right)^{\mathrm{T}}=\mathbf{0}$ and $\gamma_{j}=0$.

(ii) For $k=0,1, \ldots$, calculate:

$$
\begin{gathered}
E_{j}(x)=\gamma_{j} B_{j}(x)+B_{j+1}(x), \\
\alpha(x, t)=d(x, t)+\sum_{j=1}^{m} b_{j}^{k} E_{j}(x),
\end{gathered}
$$

(iii) Calculate $a_{2}, a_{1}, a_{0}$ in Eq. (14) and $\gamma_{j}$ by Eq. (15),

$$
\begin{aligned}
& E_{j}(x)=\gamma_{j} B_{j}(x)+B_{j+1}(x), \\
& E_{j}^{\prime}(x)=\gamma_{j} B_{j}^{\prime}(x)+B_{j+1}^{\prime}(x),
\end{aligned}
$$

(iv) Insert the above $E_{j}(x)$ and $E_{j}^{\prime}(x)$ into Eq. (22), and solve the linear system to obtain $b_{j}^{k+1}$. If the following convergence criterion for the relative norm of $\mathbf{b}^{k}$ is satisfied: 


$$
\left\|\mathbf{b}^{k+1}-\mathbf{b}^{k}\right\| \leq \varepsilon
$$

then stop the iterations, otherwise, go to (ii) to the next step. Notice that $a_{1}^{2}-4 a_{0} a_{2}$ in Eq. (15) may be negative in the first few iterations, and we use $\left|a_{1}^{2}-4 a_{0} a_{2}\right|$ to avoid the interruption of program.

\section{Numerical Tests to Recover $\alpha(x, t)$}

When the measurements of strains are contaminated by noise, we can simulate the noisy data $\bar{F}\left(t_{i}\right)$, $i=1, \ldots, n$. in Eq. (4) by adding random errors on the exact values of $F\left(t_{i}\right), i=1, \ldots, n$ by

$$
\bar{F}\left(t_{i}\right)=F\left(t_{i}\right)+s R(i), \quad i=1, \ldots, n
$$

where, $s$ is the intensity of measurement errors, assumed to be the same for all measurements, and $R(i)$ is a normally distributed random error between $[-1,1]$. In the numerical examples given below the noisy data $\bar{F}\left(t_{i}\right)$ are used as the inputs to the numerical method. We first estimate two space-dependent wave speed functions $\alpha(x)$, where $t$ is viewed as a parameter and we set it to be $t=t_{f}$. Then we estimate two space-time-dependent wave speed functions $\alpha(x, t)$, where $t$ is a time variable.

We use the relative error:

$$
\left|\frac{\alpha_{n}\left(x_{j}, t_{j}\right)-\alpha\left(x_{j}, t_{j}\right)}{\alpha\left(x_{j}, t_{j}\right)}\right|
$$

where, $\alpha_{n}\left(x_{j}, t_{j}\right)$ and $\alpha\left(x_{j}, t_{j}\right)$ are, respectively, the numerically recovered wave speed and exact wave speed at $N$ points $\left(x_{j}, t_{j}\right), j=1, \ldots, N$, to assess the accuracy of numerical solution and also consider the magnitude of $\alpha\left(x_{j}, t_{j}\right), j=1, \ldots, N$. Moreover, we consider a relative root mean-square-error defined by:

$$
e(\alpha)=\sqrt{\frac{\sum_{j=1}^{N}\left[\alpha_{n}\left(x_{j}, t_{j}\right)-\alpha\left(x_{j}, t_{j}\right)\right]^{2}}{\sum_{j=1}^{N} \alpha^{2}\left(x_{j}, t_{j}\right)}}
$$

to further assess the accuracy of numerically recovered wave speed.

\subsection{Example 1}

First, we consider

$$
\begin{gathered}
\alpha(x)=1+3 x^{2}+4 x^{4}, \\
u(x, t)=(x-3)^{2} e^{-t},
\end{gathered}
$$

and the exact $H(x, t)$ can be derived from Eq. (1).

In this identification of $\alpha(x)$ we have fixed $t_{f}=2$ and $s=20 \%$. Applying the BFM with $m=10$ and $\varepsilon=10^{-10}$, the iterative algorithm converges with three iterations as shown in Fig. 1a. Upon comparing the numerically recovered and the exact $\alpha(x)$, good result is obtained with the maximum relative error being 0.148 as shown in Fig. $1 \mathrm{~b}$, and $e(\alpha)=4.74 \times 10^{-2}$. Although only eight data on boundaries are employed to estimate $\alpha(x)$, the present result is accurate and robust against large noise.

\subsection{Example 2}

Then, we consider

$$
\begin{aligned}
& \alpha(x)=15 x^{2}+5 \exp \left[-2(x-0.5)^{2}\right], \\
& u(x, t)=(x-3)^{2} e^{-t}
\end{aligned}
$$

and the exact $H(x, t)$ can be derived from Eq. (1).

Under $t_{f}=2$ and $s=20 \%$, applying the BFM with $m=5$ and $\varepsilon=10^{-5}$ to solve this problem, the iterative algorithm converges with three iterations as shown in Fig. 2a. Upon comparing the numerically recovered wave speed with the exact $\alpha(x)$, good result is obtained with the maximum relative error being $5.7 \times 10^{-2}$ as shown in Fig. 2b, and $e(\alpha)=2.78 \times 10^{-2}$ is small.

\subsection{Example 3}

In this example we recover a space-time-dependent wave speed function, given by

$$
\begin{aligned}
& \alpha(x)=(x-3)^{2}+t^{2}, \\
& u(x, t)=(x-3)^{2} e^{-t}
\end{aligned}
$$

and the exact $H(x, t)$ can be derived from Eq. (1). 

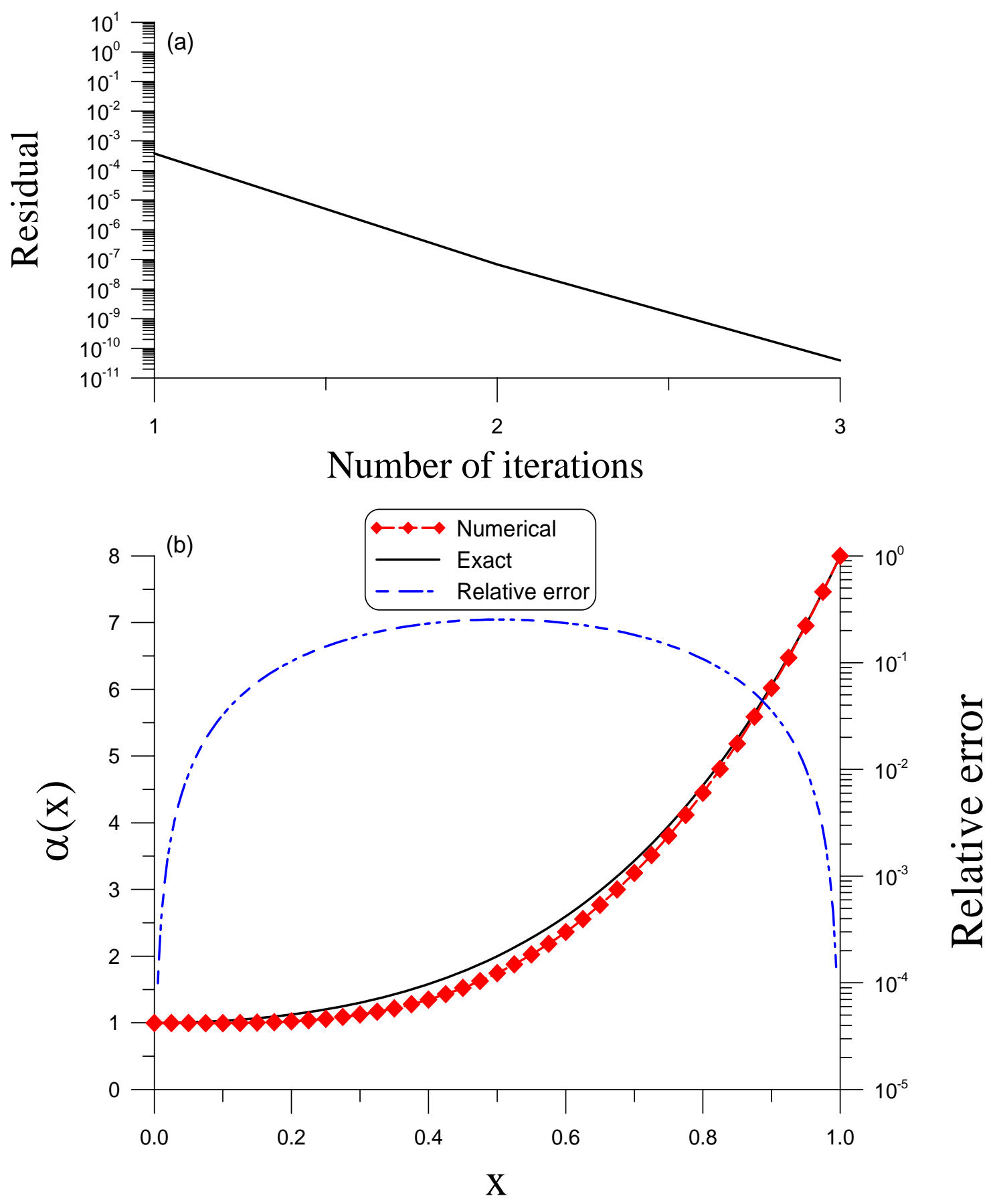

Fig. 1 For example 1 solved by the BFM iterative algorithm, (a) convergence rate, and (b) comparing numerically recovered and exact wave speeds and showing relative error. 

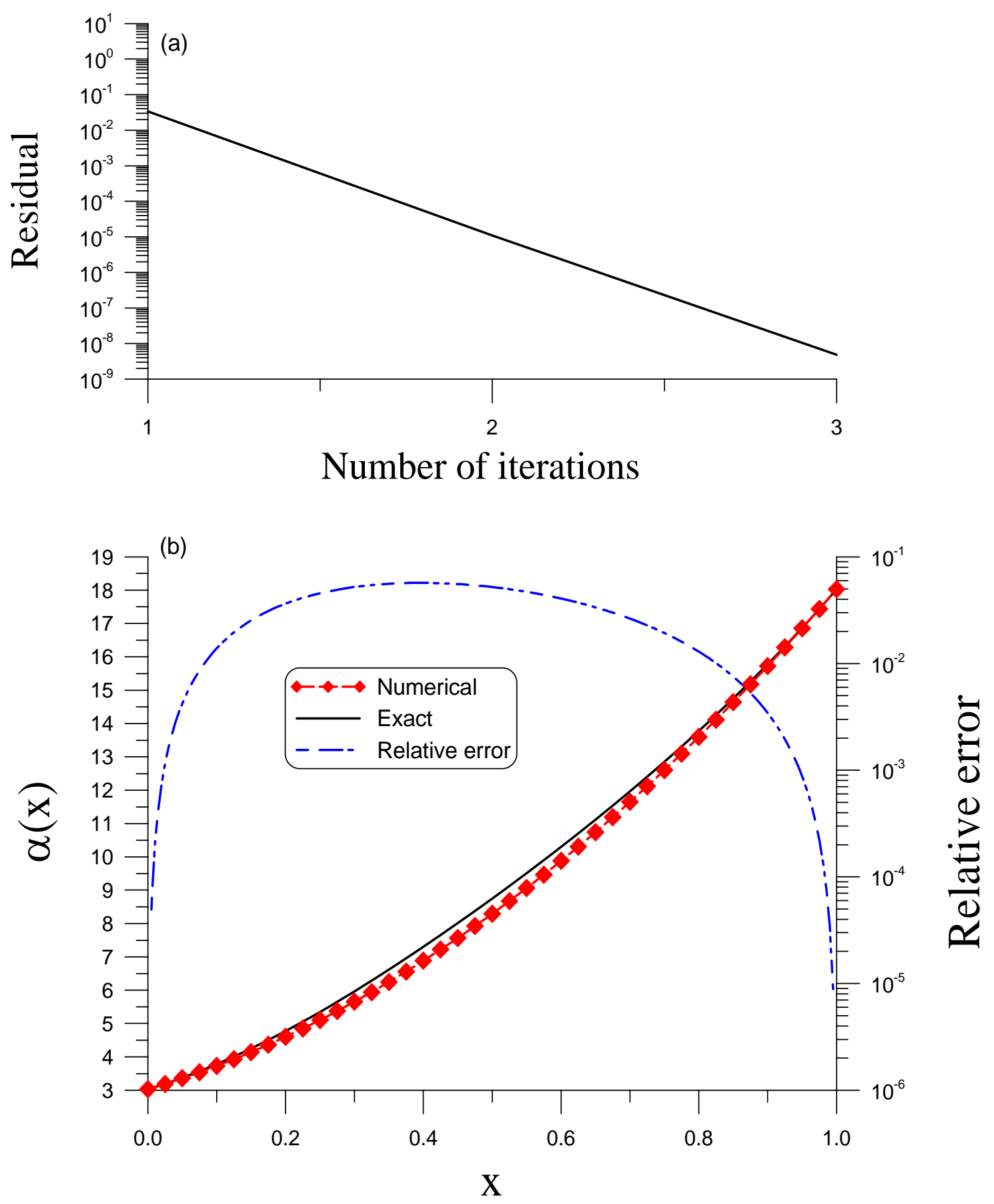

Fig. 2 For example 2 solved by the BFM iterative algorithm, (a) convergence rate, and (b) comparing numerically recovered and exact wave speeds and showing relative error. 
The noise imposed is $s=20 \%$. Applying the BFM with $m=3$ and $\varepsilon=10^{-5}$, the iterative algorithm converges with one or two iterations at each time step. Upon comparing the numerically recovered and the exact $\alpha(x, t)$, very good result is obtained with the maximum relative error over the plane $[0,1] \times(0,1]$ being $3.06 \times 10^{-4}$ as shown in Fig. 3, and $e(\alpha)=3.31 \times 10^{-5}$ is very small.

\subsection{Example 4}

In this example we recover another space-time-dependent wave speed function by

$$
\begin{aligned}
& \alpha(x)=\exp \left(1+x^{2} t^{2}+x+t\right) \\
& u(x, t)=(x-3)^{2} e^{-t}
\end{aligned}
$$

and the exact $H(x, t)$ can be derived from Eq. (1).

The noise imposed is $s=20 \%$. Applying the BFM with $m=3$ and $\varepsilon=10^{-2}$, the iterative algorithm converges with one or two iterations at each time step. Upon comparing the numerical and exact $\alpha(x, t)$ in Fig. 4 , they are close with the maximum relative error over the plane $[0,1] \times(0,1]$ being 0.28 , and $e(\alpha)=6.73 \times 10^{-2}$ is acceptable.

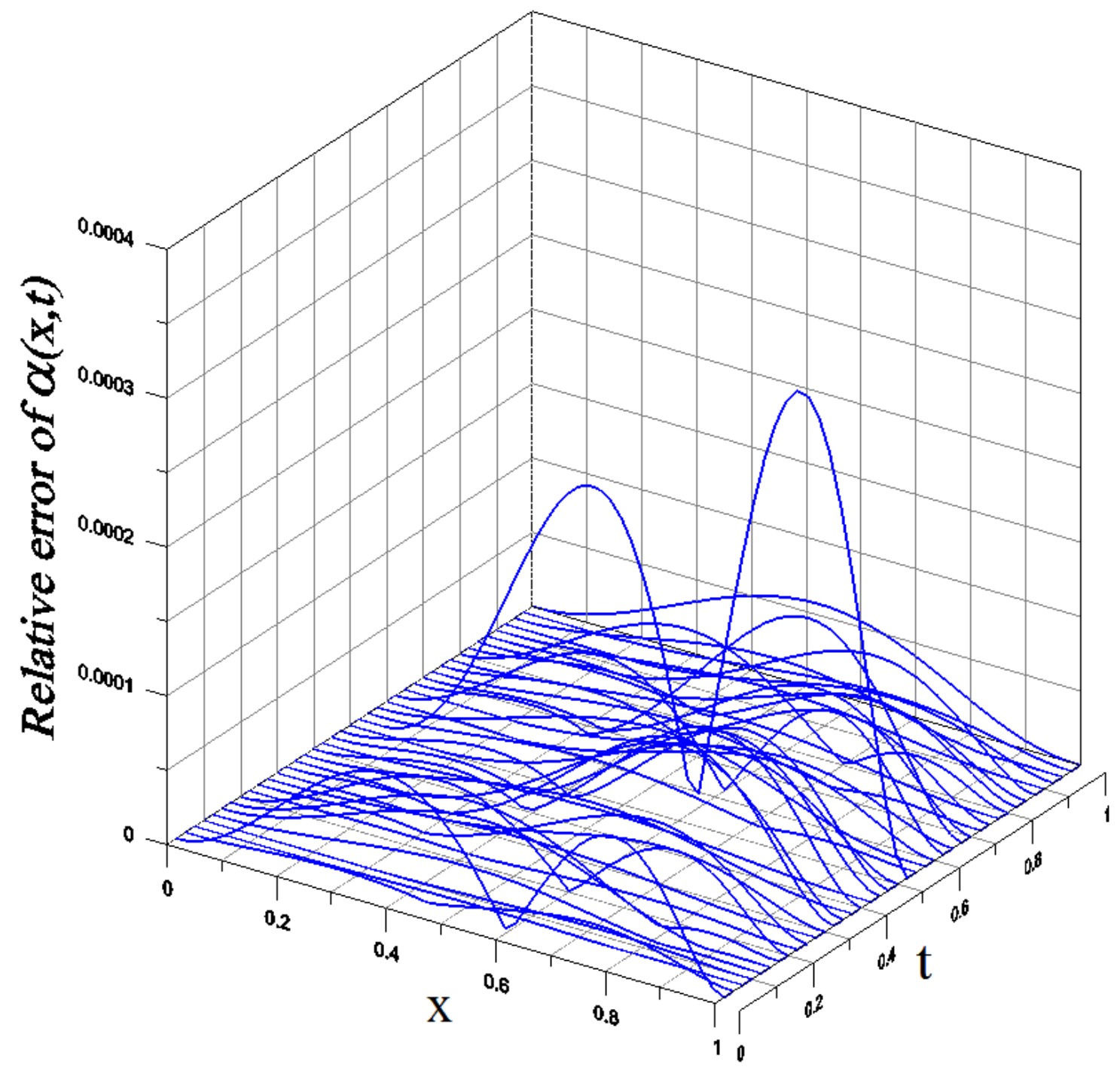

Fig. 3 For example 3 solved by the BFM iterative algorithm, showing relative error of wave speed. 


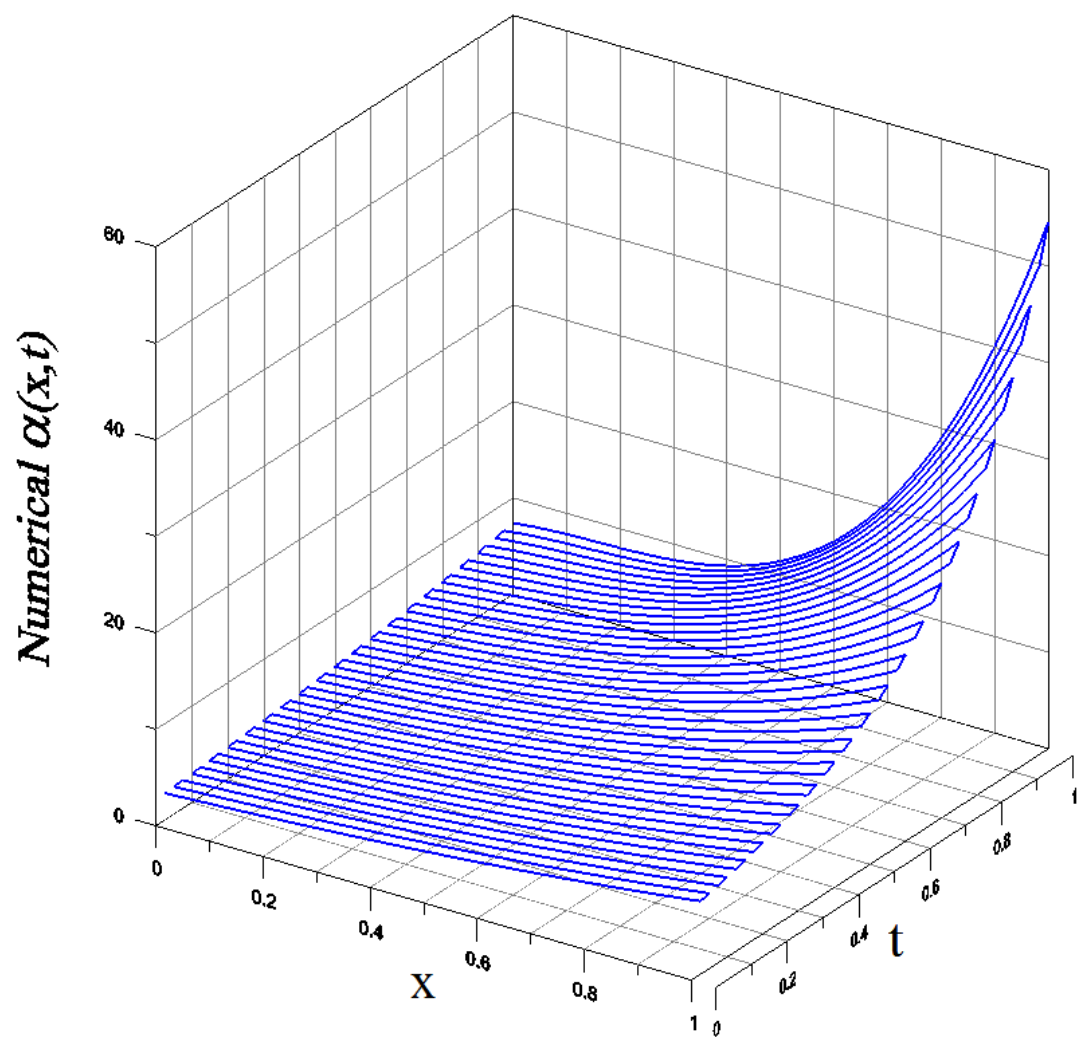

(a)

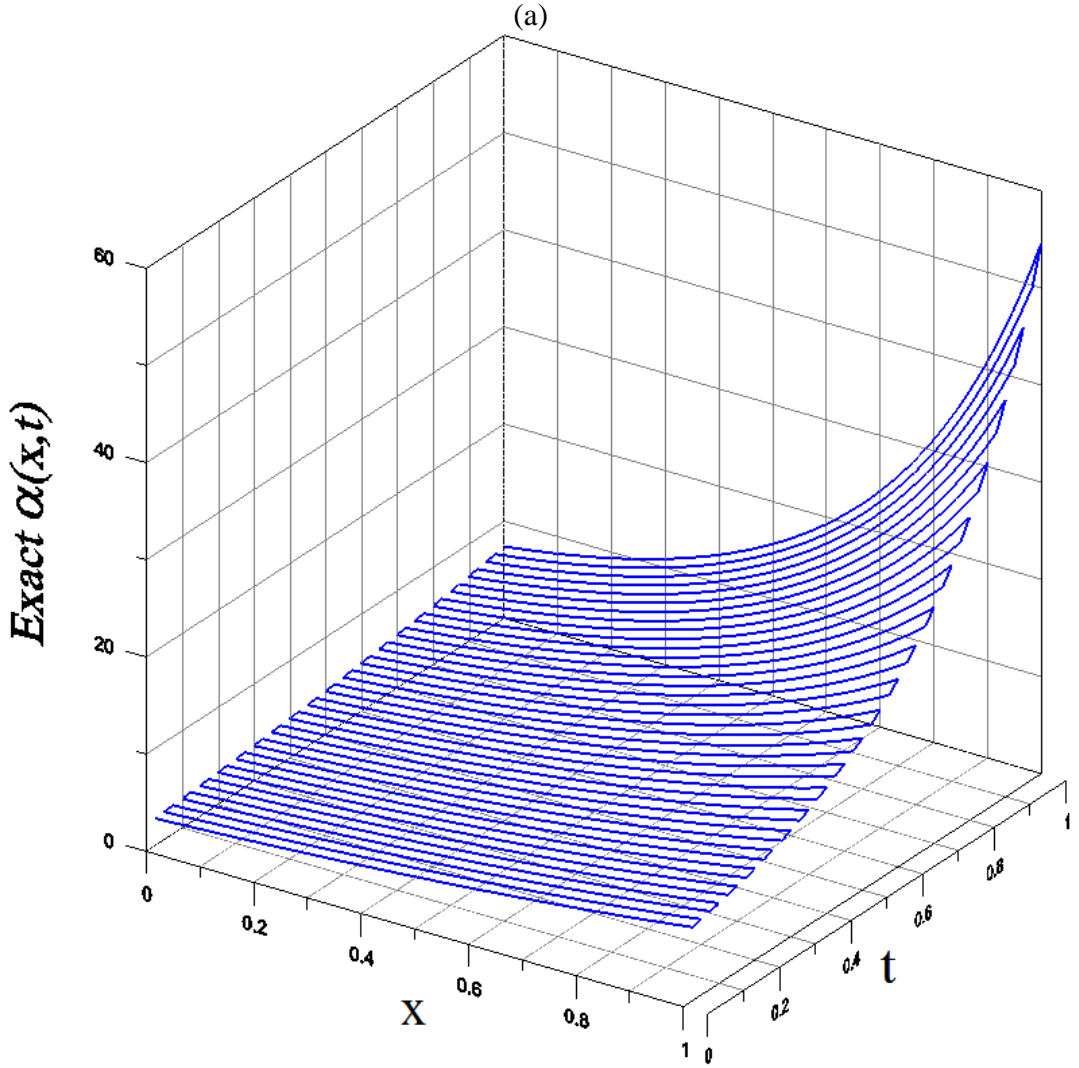

(b)

Fig. 4 For example 4 solved by the BFM iterative algorithm, comparing (a) numerically recovered and (b) exact wave speeds. 


\section{Numerical Algorithm to Recover $H(x, t)$ and Examples}

Sometimes we may encounter the problem that the wave source function in the wave equation is unknown. To resolve such an inverse wave source problem by using the control results of distributed systems was ever conducted by Yamamoto [21, 22], Bruckner and Yamamoto [23], and Yamamoto and ZHANG [24]. In higher dimensional domains the reciprocity gap functional technique leads to the reconstruction of smoother unknown sources by using the boundary observations $[17,20]$. Komornik and Yamamoto [18] have determined the positions of point sources in a one-dimensional wave equation, and later the estimation results are extended to multi-dimension in Ref. [19]. Most researchers are concerned with the determination of point sources. Liu et al. [25] have developed a differencing technique to generate a small scale linear system to recover the spatial-dependent or temporal-dependent wave sources. Liu [26] has developed a boundary integral equation method to recover the term-wise separable wave source function, and Liu [27] has developed a polynomial Trefttz method to recover the time-dependent wave source function. We will continue the BFM for the recovery of a space-time dependent wave source by using two extra strains measured on two boundaries.

\subsection{Numerical Algorithm to Recover $H(x, t)$}

In this section we attempt to recover the unknown wave source $H(x, t)$ through the boundary measurements of strains. The numerical procedure for recovering $H(x, t)$ is given in the following form to find the expansion coefficients $c_{j}$ in

$$
H(x, t)=D(x, t)+\sum_{j=1}^{m} c_{j} E_{j}(x)
$$

where,

$$
\begin{gathered}
H(x, t)=\frac{1}{\ell^{3}}\left[2 H_{0}(t)-2 H_{\ell}(t)+H_{0}^{\prime}(t) \ell+H_{\ell}^{\prime}(t) \ell\right] x^{3} \\
-\frac{1}{\ell^{2}}\left[3 H_{0}(t)-3 H_{\ell}(t)+2 H_{0}^{\prime}(t) \ell+H_{\ell}^{\prime}(t) \ell\right] x^{2}+H_{0}^{\prime}(t) x+H_{0}(t) .
\end{gathered}
$$

The data $H_{0}(t)=H(0, t), H_{\ell}(t)=H(\ell, t), H_{0}^{\prime}(t)=H^{\prime}(0, t)$ and $H_{\ell}^{\prime}(t)=H^{\prime}(\ell, t)$ are supposed to be provided.

The numerical procedures of BFM for recovering unknown wave source function are summarized as follows.

(i) Give $t$ in a time interval of $t \in\left(0, t_{f}\right]$, and give $m, \varepsilon$, and an initial guess of $\mathbf{c}^{\mathbf{0}}=\left(c_{1}, \ldots, c_{m}\right)^{\mathrm{T}}=\mathbf{0}$ and $\gamma_{j}=0$,

(ii) For $k=0,1, \ldots$, calculate:

$$
\begin{gathered}
E_{j}(x)=\gamma_{j} B_{j}(x)+B_{j+1}(x), \\
H(x, t)=D(x, t)+\sum_{j=1}^{m} c_{j}^{k} E_{j}(x), \\
a_{2}=\int_{0}^{\ell} \alpha(x, t) B_{j}^{\prime}(x)^{2} d x, \\
a_{1}=\int_{0}^{\ell}\left\{2 \alpha(x, t)\left[B_{j+1}^{\prime}+B_{0}^{\prime}(x, t)\right] B_{j}^{\prime}(x)+\left[\ddot{B}_{0}(x, t)-H(x, t)\right] B_{j}(x)\right\} d x,
\end{gathered}
$$




$$
\begin{gathered}
a_{0}=\int_{0}^{\ell}\left\{\alpha(x, t)\left[B_{j+1}^{\prime}+B_{0}^{\prime}(x, t)\right]^{2}+\left[\ddot{B}_{0}(x, t)-H(x, t)\right]\left[B_{j+1}(x)+B_{0}(x, t)\right]\right\} d x-F(t), \\
\gamma_{j}=\frac{-a_{1}-\sqrt{a_{1}^{2}-4 a_{0} a_{2}}}{2 a_{2}}, \\
E_{j}(x)=\gamma_{j} B_{j}(x)+B_{j+1}(x), \\
E_{j}^{\prime}(x)=\gamma_{j} B_{j}^{\prime}(x)+B_{j+1}^{\prime}(x),
\end{gathered}
$$

(iii) Insert the above $E_{j}(x)$ and $E_{j}^{\prime}(x)$ into

$$
\begin{gathered}
C_{i} \int_{0}^{\ell}\left[E_{j}(x)+B_{0}(x, t)\right] E_{i}(x) d x \\
=\int_{0}^{\ell}\left[E_{j}(x)+B_{0}(x, t)\right]\left[\ddot{B}_{0}(x, t)-D(x, t)\right] d x+\int_{0}^{\ell} \alpha(x, t)\left[E_{j}^{\prime}(x)+B_{0}^{\prime}(x, t)\right]^{2} d x-F(t),
\end{gathered}
$$

and solve it to obtain $c_{j}^{k+1}$. If the following convergence criterion for the relative norm of $\mathbf{c}^{k}$ is satisfied:

$$
\left\|\mathbf{c}^{k+1}-\mathbf{c}^{k}\right\| \leq \varepsilon
$$

then stop the iterations; otherwise, go to (ii) to the next step. In the first few iterations, $a_{1}^{2}-4 a_{0} a_{2}$ may be negative, and we use $\left|a_{1}^{2}-4 a_{0} a_{2}\right|$ to avoid the interruption of program.

\subsection{Example 5}

In this example we recover the space-time dependent and non-separable wave source function generated from Eq. (28), which is given by:

$$
H(x, t)=(x-3)^{2} e^{-t}+(4-2 x) \exp \left(1+x^{2} t^{2}+x\right)(31)
$$

The noise imposed is $s=20 \%$. Applying the BFM with $m=3$ and $\varepsilon=10^{-2}$, the iterative algorithm converges with one to four iterations for each time step. Upon comparing the numerical and exact $H(x, t)$ in Fig. 5, good result is obtained with the maximum relative error over the plane $[0,1] \times(0,1]$ being 0.357 , and $e(H)=7.25 \times 10^{-2}$. Notice that the maximum value of $H(x, t)$ over the plane $[0,1] \times(0,1]$ is 202.33 .

\subsection{Example 6}

In this example we recover a more complex non-separable and space-time dependent wave source function with

$$
\begin{gathered}
\alpha(x, t)=1+x^{2} t^{2}+x+t \\
u(x, t)=t+x^{2}+x^{3}+\exp \left(x^{2} t^{2}\right), \\
H(x, t)=\left(2 x+4 x^{2} t^{2}-t^{2}-2 x^{2} t^{4}-t^{4}-x^{2} t^{6}-x t^{4}-t^{5}\right) \exp \left(x t^{2}\right) \\
-\left(1+2 x^{2} t^{2}\right)\left(2 x+3 x^{2}\right)-\left(1+x^{2} t^{2}+x+t\right)(2+6 x)
\end{gathered}
$$

The noise imposed is $s=20 \%$. Applying the BFM with $m=3$ and $\varepsilon=10^{-2}$, the iterative algorithm converges with one iteration for each time increment. Upon comparing the numerically recovered wave source with the exact $H(x, t)$, good result is obtained with the maximum relative error over the plane 

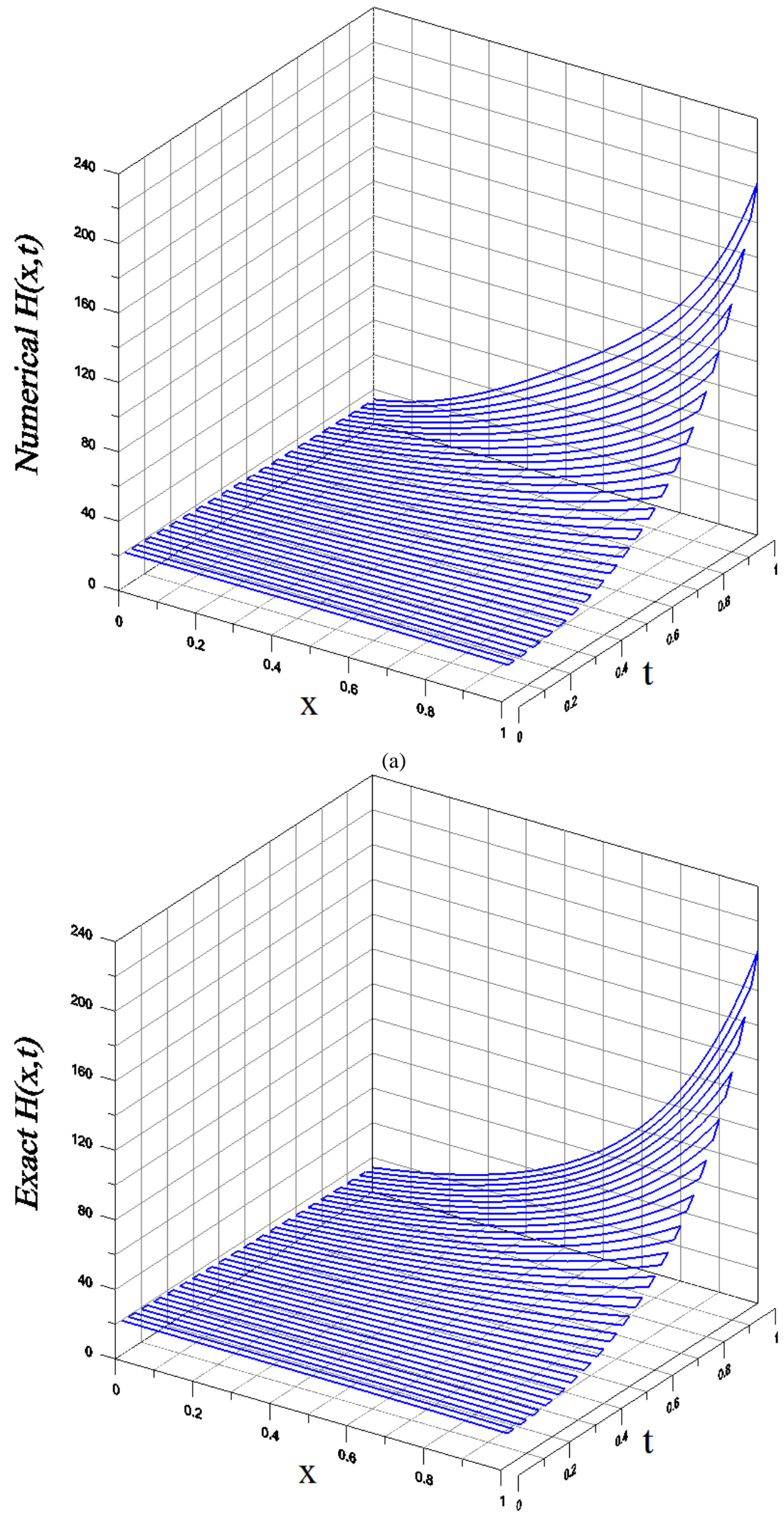

(b)

Fig. 5 For example 5 solved by the BFM iterative algorithm, comparing (a) numerically recovered and (b) exact wave sources. 


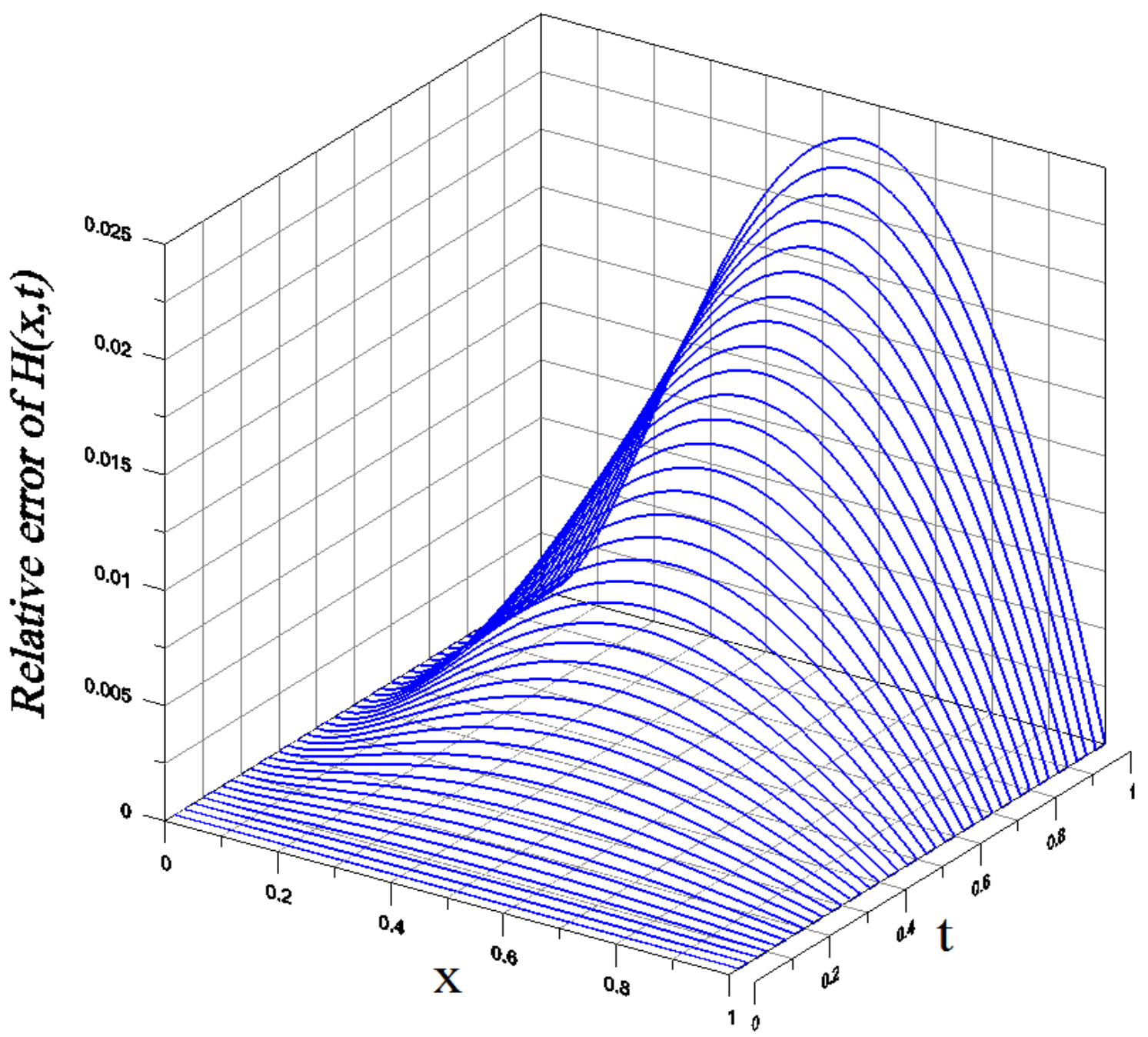

Fig. 6 For example 6 solved by the BFM iterative algorithm, showing relative error of wave source.

$[0,1] \times(0,1]$ being $2.33 \times 10^{-2}$ as shown in Fig. 6, and $e(H)=9.77 \times 10^{-3}$ is quite small. Notice that the maximum value of $H(x, t)$ over the plane $[0,1] \times(0,1]$ is 49.72 .

\subsection{Example 7}

Finally, we recover a very complex non-separable and space-time dependent wave source function generated from

$$
\begin{aligned}
& \alpha(x, t)=1+x^{2} t^{2}+x+t, \\
& u(x, t)=\exp (x+t) \cos (x t)
\end{aligned}
$$

The exact $H(x, t)$ can be obtained by inserting the above $\alpha(x, t)$ and $u(x, t)$ into Eq. (1).

The noise imposed is $s=20 \%$. Applying the BFM with $m=5$ and $\varepsilon=10^{-2}$, the iterative algorithm converges from one to sixteen iterations for each time step. Upon comparing the numerically recovered and exact $H(x, t)$ we show the error in Fig. 7, of which good result is obtained with the maximum error over the plane $[0,1] \times(0,1]$ being 3.13 and $e(H)=9.77 \times 10^{-3}$ is acceptable. Notice that the maximum absolute value of $H(x, t)$ over the plane $[0,1] \times(0,1]$ is 43.98 . 


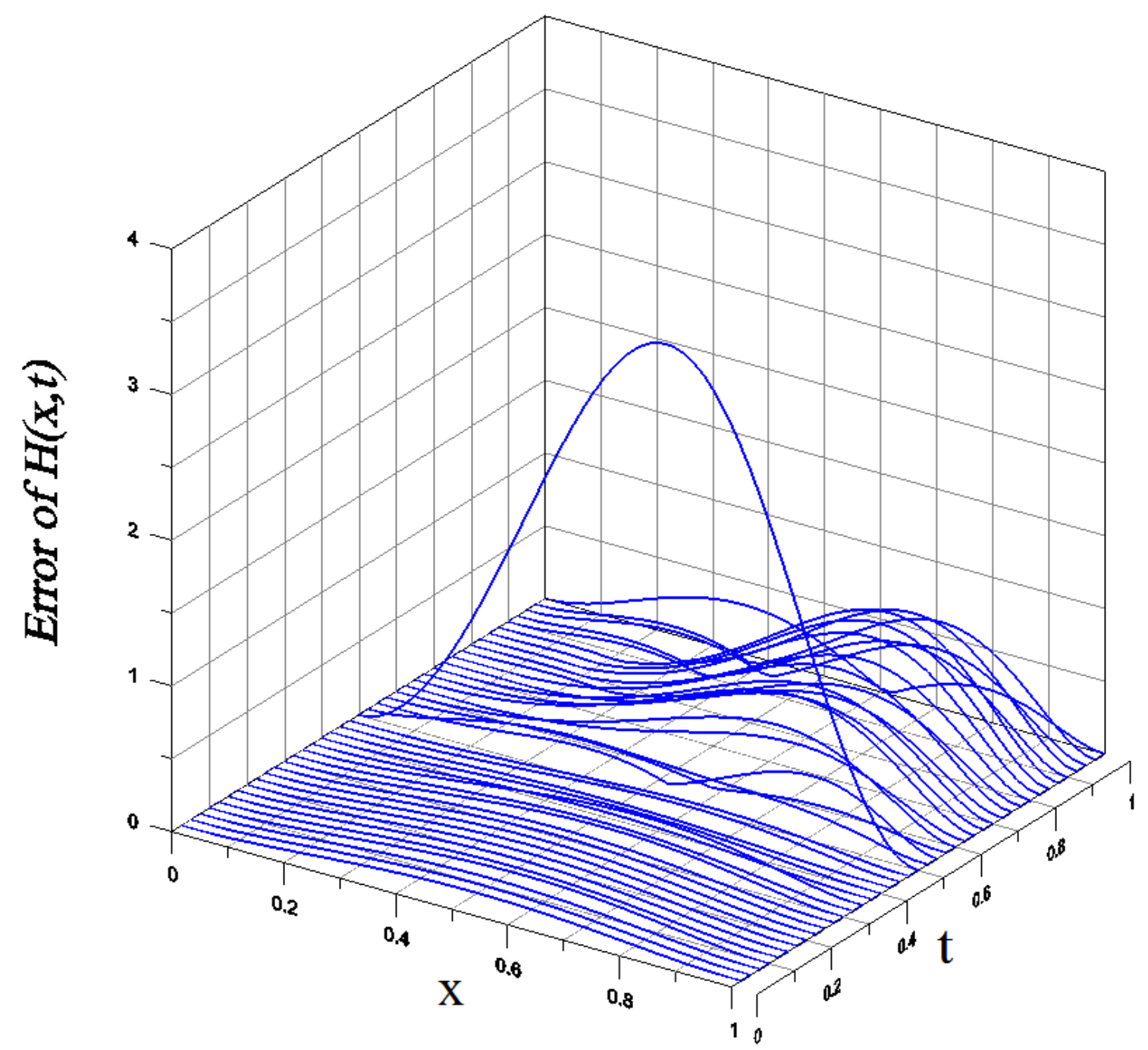

Fig. 7 For example 7 solved by the BFM iterative algorithm, showing error of wave source.

\section{Conclusions}

In this paper, a dynamic energy identity derived from the wave equation was used to set up a linear space of the energetic boundary functions, which not only satisfy the homogeneous boundary conditions but also preserve the energy in time. In terms of the energetic boundary functions as bases to expand the unknown wave speed, we have transformed the inverse coefficient problem to recover the space-time dependent wave speed into a linear system to determine the expansion coefficients of the unknown wave speed. On the other hand, we have transformed the inverse problem to recover the space-time dependent wave source function into solving a linear system to determine the expansion coefficients of the unknown function at each time step. Therefore, we can fast recover the unknown functions in the linear space, which are supplemented with extra boundary data of time-dependent strains and the boundary values of the unknown functions to be recovered. Seven examples confirmed the efficiency and accuracy of the presented BFMs, of which the convergence is only through a few iterations at each time step. To the best knowledge of authors, in the literature there exists no such a similar work which can recover the space-time dependent wave speed and wave source functions only through the boundary 
measurements of strains, without needing to know the initial conditions and internal over-specified conditions of $u(x, t)$.

\section{Acknowledgements}

The Thousand Talents Plan of China under the Grant Number A1211010 and the Fundamental Research Funds for the Central Universities under the Grant Number 2017B05714 for the financial support to the first author are highly appreciated.

\section{References}

[1] Lin, J., Chen, C. S., Liu, C. S., and Lu, J. 2016. "Fast Simulation of Multi-dimensional Wave Problems by the Sparse Scheme of the Method of Fundamental Solutions." Computers \& Mathematics with Applications 73 (3): 555-67.

[2] Liu, C. S., and Kuo, C. L. 2016. “A Multiple-Direction Trefftz Method for Solving the Multi-dimensional Wave Equation in an Arbitrary Spatial Domain.” Journal of Computational Physics 321: 39-54.

[3] Baev, A. V. 1988. "One Formulation of the Inverse Boundary-Value Problem for the Wave Equation and an Iterative Solution Method.” Dokl. Akad. Nauk SSSR 287 (4): 818-21.

[4] Baev, A. V. 1987. “An Iterative Solution Method for One Inverse Problem for the Wave Equation on an Interval.” Zh. Vychisl. Mat. Mat. Fiz. 27: 1022-31.

[5] Baev, A. V. 1988. "A Solution Method for the Inverse Scattering Problem for the Wave Equation.” Zh. Vychisl. Mat. Mat. Fiz. 28: 25-33.

[6] Tadi, M. 1997. "Explicit Method for Inverse Wave Scattering in Solids.” Inverse Problems 13 (2): 509-21.

[7] Tadi, M. 1998. "Evaluation of the Elastic Property Based on Boundary Measurement.” Acta Mechanica 129 (3-4): 231-41.

[8] Tadi, M. 1999. "Inverse Wave Scattering in 2D Elastic Solids.” Acta Mechanica 136 (1-2): 1-15.

[9] Na, S. W., and Kallivokas, L. F. 2009. "Direct Time-Domain Soil Profile Reconstruction for 1D Semi-infinite Domains.” Soil Dynamics and Earthquake Engineering 29 (6): 1016-26.

[10] Beilina, L., and Klibanov, M. V. 2008. “A Globally Convergent Numerical Method for a Coefficient Inverse Problem.” SIAM Journal on Scientific Computing 31 (1): 478-509.

[11] Liao, W. 2011. “A Computational Method to Estimate the Unknown Coefficient in a Wave Equation Using Boundary Measurements.” Inverse Problems in Science and Engineering 19 (6): 855-77.

[12] Goncharskii, A. V., and Romanov, S. Y. 2012. "Two Approaches to the Solution of Coefficient Inverse Problems for Wave Equations.” Computational Mathematics and Mathematical Physics 52 (2): 478-509.

[13] Liu, C. S. 2010. "A Lie-Group Adaptive Method for Imaging a Space-Dependent Rigidity Coefficient in an Inverse Scattering Problem of Wave Propagation." CMC-Computers Materials \& Continua 18 (1): 1-21.

[14] Ismailov, M. I., and Tekin, I. 2016. "Inverse Coefficient Problems for a First Order Hyperbolic System.” Applied Numerical Mathematics 106: 98-115.

[15] Rashedi, K., Adibi, H., and Dehghan, M. 2013. "Application of the Ritz-Galerkin Method for Recovering the Space Wise-Coefficients in the Wave Equation." Computers \& Mathematics with Applications 65 (12): 1990-2008.

[16] Jang, T. S., Choi, H. S., and Kinoshita, T. 2000. "Solution of an Unstable Inverse Problem: Wave Source Evaluation from Observation of Velocity Distribution.” Journal of Marine Science and Technology 5 (4): 1990-2008.

[17] El Badia, A., and Ha-Duong, T. 2001. "Determination of Point Wave Sources by Boundary Measurements." Journal of Marine Science and Technology 17 (4): 1127-39.

[18] Komornik, V., and Yamamoto, M. 2002. "Upper and Lower Estimates in Determining Point Sources in a Wave Equation.” Inverse Problems 18 (4): 319-29.

[19] Komornik, V., and Yamamoto, M. 2002. "Estimation of Point Sources and Applications to Inverse Problems." Inverse Problems 21 (6): 2051-70.

[20] Ohe, T., Inui, H., and Ohnaka, K. 2011. "Estimation of Point Sources and Applications to Inverse Problems.” Inverse Problems 27 (11): 115011.

[21] Yamamoto, M. 1995. "Stability, Reconstruction and Regularization for an Inverse Source Hyperbolic Problem by a Control Method.” Inverse Problems 11 (2): 481-96.

[22] Yamamoto, M. 1996. "Determination of Forces in Vibrations of Beams and Plates by Point Wise and Line Observations." Journal of Inverse and Ill-Posed Problems 4 (5): 437-57.

[23] Bruckner, G., and Yamamoto, M. 2000. "Determination of Point Wave Sources by Pointwise Observations: Stability and Reconstruction.” Inverse Problems 16 (3): 115011.

[24] Yamamoto, M., and ZHANG, X. 2001. "Global Uniqueness and Stability for an Inverse Wave Source Problem for Less Regular Data.” Journal of Mathematical Analysis and Applications 263 (2): 479-500.

[25] Liu, C. S., Chen, W., and Lin, J. 2016. “A Homogenized Function to Recover Wave Source by Solving a Small Scale Linear System of Differencing Equations.” Journal 
of Mathematical Analysis and Applications 111 (5): 421-35.

[26] Liu, C. S. 2017. “A Global Domain/Boundary Integral Equation Method for the Inverse Wave Source and Backward Wave Problems.” Inverse Problems in Science and Engineering 25 (4): 506-31.

[27] Liu, C. S. 2017. "The Polynomial Trefftz Method for Solving Backward and Inverse Source Wave Problems.” Journal of Computational and Applied Mathematics 321: 211-25. 